\title{
Investigation of the Growth Mechanism of Iron Oxide Nanoparticles via a Seed-Mediated Method and Its Cytotoxicity Studies
}

\author{
Jing- Hong Huang, ${ }^{\dagger}$ Harshala J. Parab, ${ }^{\dagger, \|}$ Ru-Shi Liu,,${ }^{\dagger}{ }^{\dagger}$ Tsung-Ching Lai, ${ }^{\ddagger}$ Michael Hsiao, \\ Chung-Hsuan Chen, ${ }^{\ddagger}$ Hwo-Shuenn Sheu, ${ }^{\S}$ Jin-Ming Chen, ${ }^{\S}$ Din-Ping Tsai, ${ }^{\perp}$ and \\ Yeu-Kuang Hwu" \\ Department of Chemistry, National Taiwan University, Taipei 106, Taiwan, The Genomics Research Center, \\ Academia Sinica, Taipei 115, Taiwan, National Synchrotron Radiation Research Center, Hsinchu 300, Taiwan, \\ Department of Physics, National Taiwan University, Taipei 106, Taiwan, and Institute of Physics, Academia \\ Sinica, Taipei 115, Taiwan
}

Received: April 21, 2008; Revised Manuscript Received: July 15, 2008

\begin{abstract}
We report here the development of a seed-mediated growth method for the synthesis of iron oxide nanoparticles with tunable size distribution and magnetic properties. We have investigated the size distribution of seed as well as iron oxide nanoparticles during the growth process using transmission electron microscopy (TEM). It has been observed that the distribution of size gradually becomes narrow with time via the intraparticle ripening process and Oswald ripening process. The magnetization measurements were performed using a superconducting quantum interference device (SQUID) from room temperature to $5 \mathrm{~K}$ to determine the effect of size distribution on the blocking temperature $\left(T_{\mathrm{b}}\right)$ and splitting temperature $\left(T_{\text {splitting }}\right)$. The thermogravimetric analyses exhibited the size-dependent weight loss of the magnetic nanoparticles. The in vitro cytotoxicity tests were also performed to determine the cell viability as a function of size and concentration of the magnetic nanoparticles.
\end{abstract}

\section{Introduction}

The iron oxide nanoparticles have been extensively studied for medical imaging, drug targeting, biomolecular separation, and catalytic applications owing to their unique electrical, magnetic, and chemical properties. ${ }^{1-7}$ It is really important to obtain monodispersity during the fabrication of the nanoparticles to increase the sensitivity of these particles for the various applications. Generally, the synthesis of magnetic nanoparticles by using methods such as chemical coprecipitation ${ }^{8,9}$ and hydrothermal treatment ${ }^{10,11}$ produces broad distribution of particle sizes. Hence, the development of novel methods has been the focus of recent research to fabricate uniform and highly monodispersed nanocrystals by the thermal decomposition method. ${ }^{12-15}$ However, there are not many reports on detailed studies of the growth process of monodispersed superparamagnetic iron oxide nanoparticles (SPIONs), although the syntheses of relatively uniform maghemite and magnetite nanoparticles have been reported. ${ }^{16,17}$

Herein, we report the development of a seed-mediated growth method for the synthesis of iron oxide nanoparticles with tunable size distribution and magnetic properties. A simple method has been illustrated to fabricate the monodispersed iron oxide nanoparticles from 5 to $10 \mathrm{~nm}$ by varying the experimental parameters. It was observed that the seed-mediated growth method for iron oxide nanoparticles is advantageous since it is a noninjection or heating-up method with an easily controllable growth process. ${ }^{18,19}$ The objective of the present investigation

* To whom correspondence should be addressed. E-mail: rsliu@ntu.edu.tw. Phone: +886-2-33661169. Fax: +886-2-2363-6359.

$\dagger$ Department of Chemistry, National Taiwan University.

"Institute of Physics, Academia Sinica.

* The Genomics Research Center, Academia Sinica.

$\S$ National Synchrotron Radiation Research Center.

${ }^{\perp}$ Department of Physics, National Taiwan University. is to study the size distribution of the seed as well as the iron oxide nanoparticles during the growth process since it is not studied in detail. The iron oxide nanoparticles were characterized by X-ray diffraction (XRD), thermogravimetric analysis (TGA), $\mathrm{X}$-ray absorption spectroscopy (XAS), X-ray magnetic circular dichroism (XMCD), and superconducting quantum interference device (SQUID) measurements. The synthesis of iron oxide nanoparticles was found to be reproducible with the tunable properties. The in vitro cell viability tests were also performed for these iron oxide nanoparticles to determine their cytotoxic effects.

\section{Experimental Section}

Synthesis of Iron Oxide Nanoparticle Seeds. The seeds were prepared by following a procedure reported in the literature ${ }^{14}$ Briefly, $0.7 \mathrm{~g}$ of $\mathrm{Fe}(\mathrm{acac})_{3}(2 \mathrm{mmol}), 1.6 \mathrm{~g}$ of oleic acid $(6 \mathrm{mmol}), 1.6 \mathrm{~g}$ of oleylamine $(6 \mathrm{mmol}), 2.3 \mathrm{~g}$ of 1,2tetradecanediol $(10 \mathrm{mmol})$, and $20 \mathrm{~mL}$ of phenyl ether were mixed and magnetically stirred in a Schlenk flask under a flow of nitrogen. The mixture was slowly heated to reflux for 30 min at $265{ }^{\circ} \mathrm{C}$ followed by cooling to room temperature. The nanoparticles were then dispersed in ethanol and collected in solid form after centrifugation at $9000 \mathrm{rpm}$. The product was further washed several times by hexane to remove the residual solvent. Finally, $5.4 \mathrm{~nm}$ iron oxide nanoparticles were obtained after drying in the oven, which were redispersed into hexane.

Synthesis of Different Sized Iron Oxide Nanoparticles in Growth Solution. The synthesis of iron oxide nanoparticles of different sizes involved an initial formation of iron oxide nanoparticles as seeds and subsequent growth of the particles in the presence of seeds at high temperature. The concentrations of growth solutions prepared by adjusting the concentration of $\mathrm{Fe}(\mathrm{acac})_{3}$ and phenyl ether/benzyl ether were $0.028,0.056$, $0.084,0.112 \mathrm{M}$. The mixture of $0.08 \mathrm{~g}$ of seeds and $20 \mathrm{~mL}$ of 
growth solution in the presence of 1,2-tetradecanediol, oleic acid, and oleylamine was heated to $265{ }^{\circ} \mathrm{C}$ at a rate of $4 \mathrm{deg} / \mathrm{min}$ under nitrogen atmosphere and then was kept at this temperature for 30 to $90 \mathrm{~min}$ for complete growth of nanoparticles. After the growth of the nanoparticles, the reaction mixture was cooled to room temperature followed by the addition of ethanol. The brown precipitate thus formed was redispersed into hexane.

Cytotoxicity Assay. Considering the increasing applications of the magnetic nanoparticles in the biomedical fields, the in vitro cell viability studies were performed in the presence of the magnetite nanoparticles synthesized by the above method. ${ }^{20-23}$ The normal breast epithelial cell (H184B5F5/M10) and the three types of breast cancer cells (SKBR3, MB157 and T47D) were used for cytotoxicity tests. These cells (2000 cells per $90 \mu \mathrm{L})$ with $10 \mu \mathrm{L}$ of different concentrations of nanoparticles (in 0.1 , $1,10,100 \mu \mathrm{g} / \mathrm{mL}$ ) were seeded into different wells and were incubated for $72 \mathrm{~h}$ followed by the addition of $20 \mathrm{~mL}$ of MTS (3-(4,5-dimethylthiazol-2-yl)-5-(3-carboxymethoxyphenyl)-2-(4sulfophenyl)- $2 \mathrm{H}$ - tetrazolium, inner salt) in each well. The optical density (OD) of the resulting solutions was determined $(\lambda=490 \mathrm{~nm})$ by using a microplate absorbance reader (SpectraMAX 340pc, Molecular Devices, CA).

Characterization. The morphology of magnetic nanoparticles has been analyzed by using transmission electron microscopic analysis with a field emission gun operating at $100 \mathrm{keV}$ (Hitachi H-7000). The X-ray diffraction patterns, X-ray absorption spectra (XAS), and X- ray magnetic circular dichrism spectra (XMCD) were recorded with use of the light source of BL01C2 $(\lambda=0.7749 \AA)$, BL20A, and BL11A at the National Synchrotron Radiation Research Center (NSRRC) in Taiwan. The XMCD measurements were carried out by using a $6 \mathrm{~m}$ Spherical Grating Monochromator (SGM) with a spherical Horizorital Focusing Mirror (HFM), Vertical Focusing Mirror (VFM), and toroidal Refocusing mirror (RFM) system. The samples were exposed to the synchrotron radiation from the bending magnet of the $1.5 \mathrm{GeV}$ storage ring and the magnetic field. The magnetic field strength used for the XMCD measurements was $\pm 1 \mathrm{~T}$ obtained using the current of $\pm 50 \mathrm{~A}$ under the polarized $\mathrm{X}$-rays. The magnetic properties of iron oxide nanoparticles were measured by using a superconducting quantum interference device (SQUID; Quantum Design MPMS-XL7) under 100 Oe magnetic field. The composition of nanoparticles was determined by thermogravimetric analysis (TGA; Dynamic TGA 2950).

\section{Results and Discussion}

In seed-mediated growth method, the nanoparticle formation involves two steps mainly the formation of monodispersed seeds and the growth of seed solution in the presence of both surfactants and precursor under refluxing temperature for different time intervals. To understand the growth process of monodispersed nanoparticles, TEM images were obtained at various growth times. TEM images of iron oxide nanoparticles for time intervals of 30-90 min are shown in Figure 1, panels $\mathrm{A}-\mathrm{D}$. The as-prepared seed nanoparticles showed the average size of $5.4 \mathrm{~nm}$ with a narrow size distribution. After $30 \mathrm{~min}$ of refluxing, it was observed that the size distribution became broad but the majority of nanoparticles still possessed the average size of $5.4 \mathrm{~nm}$, which is shown in Figure 1, panels B and F. The major size distribution shifted to $8 \mathrm{~nm}$ after $60 \mathrm{~min}$ as shown in Figure 1, panels $\mathrm{C}$ and $\mathrm{G}$. When the growth time was prolonged to $90 \mathrm{~min}$, the size distribution became narrow as shown in Figure 1, panels D and H. Surprisingly, after 90 min, the particles do not grow further and finally the size of the particles was "focused" at around $8 \mathrm{~nm}$. If the monomers were not consumed completely or did not form dynamic equilibrium, then the particle size would have grown further to cause particle size distribution shift to a larger one. In that case, the final size of the particles would not have been "focused" around $8 \mathrm{~nm}$. Thus from the observations, it is expected that the monomers have already been exhausted or formed dynamic equilibrium between dissolution and recrystallization. The schematic of the mechanism for the seed growth process has been shown in Figure 1. In Stage I, it was observed that the nucleation of monomer (individual red balls) takes place rapidly within the first $30 \mathrm{~min}$ (called the primary nucleation represented by aggregated red balls). The primary nucleation was followed by secondary nucleation on the surface of as-prepared seed nanoparticles, which gives rise to secondary nuclei shown as green balls in Figure 1. According to the classic Ostwald ripening process, ${ }^{24-26}$ the size distribution will be "defocusing" and continuously grow by dissolving the small particles after the monomer has been consumed completely. In Stage II, the seedmediated growth is not consistent with this part of the classic Ostwald ripening process, and it appears that the features are consistent with a ripening process between the particles (called the intraparticle ripening process). ${ }^{27-29}$ According to the concentration-dependent critical diameter, ${ }^{26}$ the smaller sized particles formed via primary nucleation below the critical diameter will be dissolved easily into the solution. Although the kinetics of the Stage II mechanism is difficult to study, the dissolution and growth can be more readily examined by eq 1 . At a dynamic equilibrium of monomer concentration, the sizedependent solubility can be developed by using the GibbsThomson equation ${ }^{30}$ considering that diffusion is the major contribution to these stages.

$$
S_{\mathrm{r}}=S_{\mathrm{b}} \exp \left(2 \sigma V_{\mathrm{m}} / r R T\right)
$$

where, $S_{\mathrm{r}}$ is the solubility of the nanoparticle, $S_{\mathrm{b}}$ is the solubility of the corresponding bulk solid, $\sigma$ is the specific surface energy, $r$ is the radius of the nanoparticle, $V_{\mathrm{m}}$ is the molar volume of the nanoparticle, $R$ is the gas constant, and $T$ is the absolute temperature. The nanoparticle solubility is inversely proportional to the exponential radius. In other words, the small particles with a high amount of surface atoms easily release the unstable surface atoms into solution by forming a complex with excess oleate ligand. The growth volume of nanoparticles can be given by following equation:

$$
\Delta V={ }^{4} /{ }_{3} \pi\left(r^{3}-r^{3}\right)=\text { constant }
$$

where, $\Delta V$ is the growth volume of nanoparticles, $r$ is the radius of nanoparticles, and $r^{\prime}$ is the radius of nanoparticles after growing. The same number of monomers diffuse into the diffusion layer of each growth particle giving constant growth volume to different sizes of particles and cause the higher growth rate for small radius particles than larger ones. ${ }^{27}$ For the growth of nanoparticles in Stage III, the intraparticle ripening process also decreases the amount of larger nanoparticles $(>10$ $\mathrm{nm}$ ) via the mass transformation process (occurred between the different sizes of green balls in Stage III). Hence, it involves two kinds of processes: (1) The particles with asize lower than critical diameter are readily dissolved into solution as monomers, whereas the growth for particles with a size above the critical diameter is strongly size-dependent and occurs via seeding on monomers. (2) The intraparticle ripening process cause a major distribution of the nanoparticles focused around $8 \mathrm{~nm}$ and does not grow much further. The results showed that the sizedependent growth rate of particles above the critical diameter and the intraparticle ripening caused the sizes of nanoparticles 

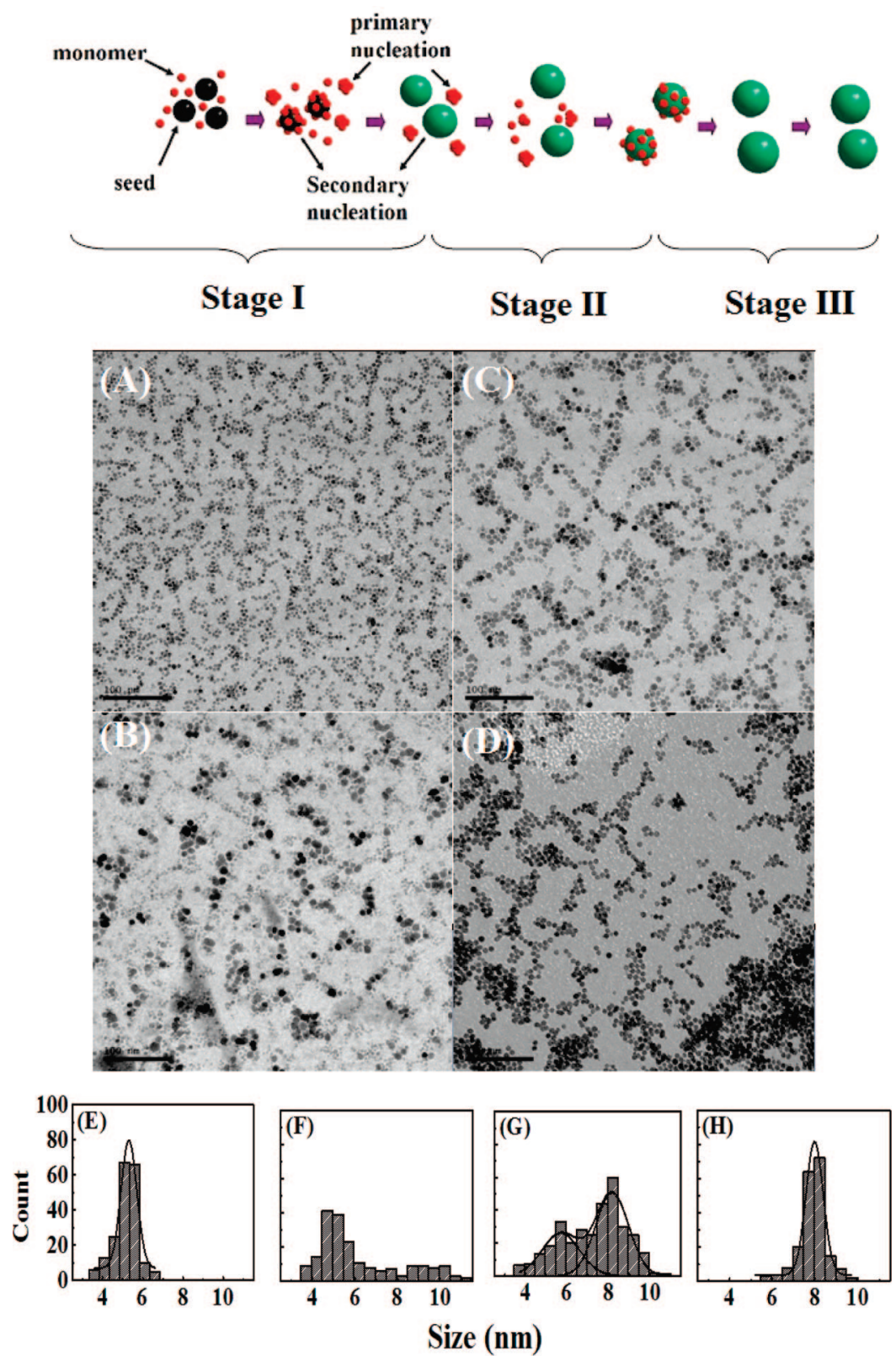

Figure 1. TEM bright field images and schematic illustrations of growth particles in $0.084 \mathrm{M}$ solution after (A) 0, (B) 30, (C) 60, and (D) 90 min, and their histograms of size distributions are given in panels $\mathrm{E}, \mathrm{F}, \mathrm{G}$, and $\mathrm{H}$, respectively.

to be "focused" around $8 \mathrm{~nm}$. In the beginning, the primary nucleation of nanoparticles may block the growth of seed and prevent seed from rapid random growth. Hence, the monomers consumed by forming small particles offer a moderate surrounding for growing particles, and then the formed small particles play the role of monomer supporter after monomers are consumed or formed dynamic equilibrium. The understanding of the mechanism supports the powerful method to obtain high-quality monodispersed iron oxide nanoparticles formed via small particles and also the ease of the method to control the growth scale in the nanometer range.

Figure 2 shows the standard deviation $(\sigma)$ of nanoparticles size distribution after refluxing for different time scales. In the beginning, the $\sigma$ value increases with increasing refluxing time owing to the result of size difference between the small nuclei and the growth particles. The maximum value has been observed between 30 and $60 \mathrm{~min}$ and then it begins to drop and deviates from the original trend. As a result the number of small particles decreases and the distribution of particle size starts to focus as

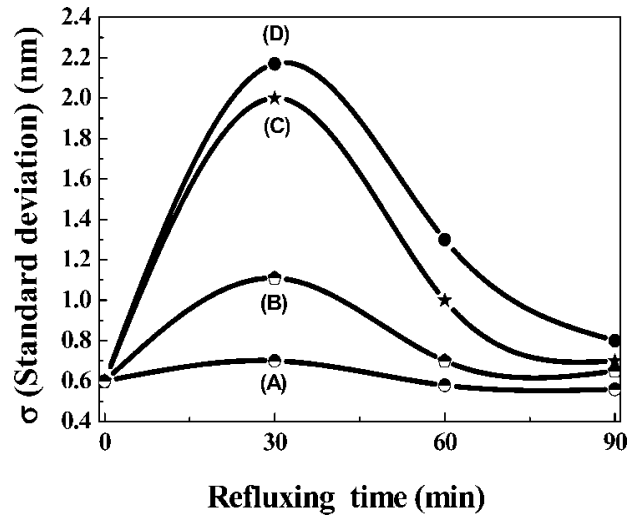

Figure 2. The standard deviation in size distribution in (A) 0.028 , (B) 0.056 , (C) 0.084 , and (D) $0.112 \mathrm{M}$ growth solution.

described previously. The rapidly decreasing $\sigma$ value in high concentration solution shows the small particles dissolve easily due to the relatively large critical radius. By controlling the 

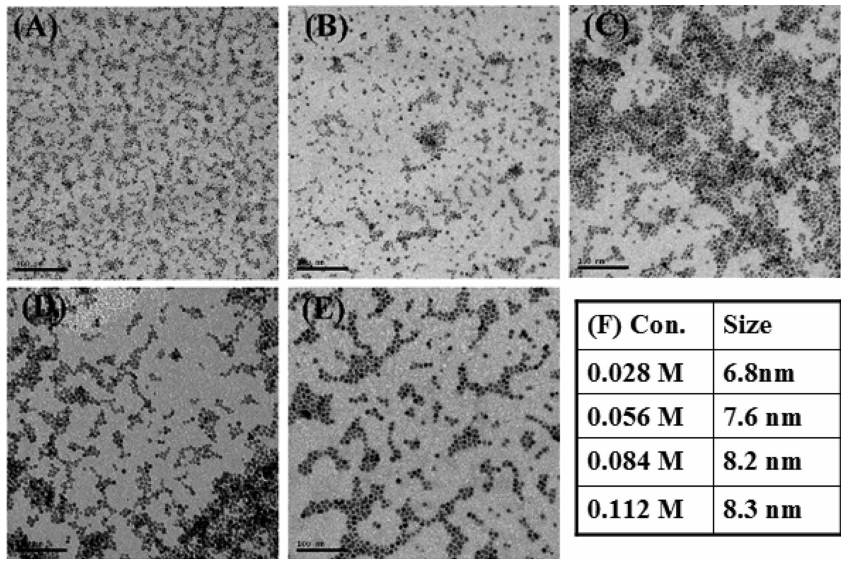

\begin{tabular}{|l|l|}
\hline (F) Con. & Size \\
\hline $0.028 \mathrm{M}$ & $6.8 \mathrm{~nm}$ \\
\hline $0.056 \mathrm{M}$ & $7.6 \mathrm{~nm}$ \\
\hline $0.084 \mathrm{M}$ & $8.2 \mathrm{~nm}$ \\
\hline $0.112 \mathrm{M}$ & $8.3 \mathrm{~nm}$ \\
\hline
\end{tabular}

Figure 3. TEM images of seed solution (A) and iron oxide nanoparticles after growth in different concentrations of growth solution such as (B) 0.028, (C) 0.056, (D) 0.084, and (E) $0.112 \mathrm{M}$, and (F) final particle size of iron oxide nanoparticles in different concentrations of growth solution.

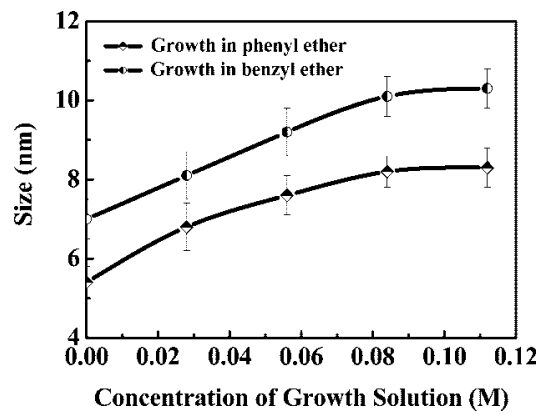

Figure 4. The concentration-dependent seed growth in phenyl ether and benzyl ether.

reaction time and various concentrations, it was possible to produce monodispersed nanoparticles with particle sizes of 6.8, $7.6,8.2$, and $8.3 \mathrm{~nm}$. The TEM images of the particles along with the seed are shown in Figure 3. Panel F in Figure 3 is the table of calculated average sizes of the particles, using the corresponding TEM images of the samples.

Figure 4 shows the concentration-dependent seed growth after reacting for $90 \mathrm{~min}$ in different solvents. The boiling temperature of benzyl ether is higher than that of phenyl ether. It was observed that the size of as-prepared seeds in benzyl ether is larger than that in phenyl ether, because the amount of monomers induced by thermal energy increases with temperature. The growth curve finally reached the saturation value due to the high concentration of solvents. The maximum value was observed at around 8.2 and $10.2 \mathrm{~nm}$ for phenyl ether and benzyl ether, respectively. Although the higher concentration causes faster seed growth, some portion of the monomers also form small particles and grow over the level of critical radius to compete with as-prepared seeds. Hence, the final particle concentration is contributed partially from primary nucleation of the particles, so that the particle size is limited. Thus, by tuning the parameters such as temperature and concentration, the series of monodispersed nanoparticles in the range of 5.4 to $10.2 \mathrm{~nm}$ are synthesized successfully.

Figure 5 shows the XRD patterns of iron oxide nanocrystals of 5.4, 6.8, 7.6, and $8.2 \mathrm{~nm}$ and the standard $\mathrm{Fe}_{3} \mathrm{O}_{4}$ and $\alpha-\mathrm{Fe}_{2} \mathrm{O}_{3}$. The peak positions and relative intensities of nanoparticles agree well with XRD patterns of standard $\mathrm{Fe}_{3} \mathrm{O}_{4}$, which confirms the well-known inverse spinel structure of the magnetite materials. The average sizes of the iron oxide nanoparticles are deduced from Sherrer's equation as shown in Table 1, which is consistent

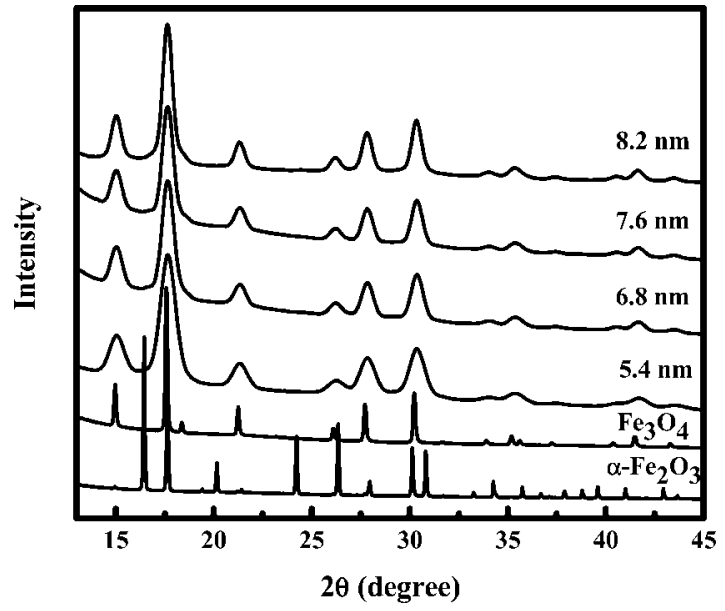

Figure 5. XRD patterns of iron oxide nanocrystals of 5.4, 6.8, 7.6, and $8.2 \mathrm{~nm}$ sizes and the $\mathrm{Fe}_{3} \mathrm{O}_{4}$ and $\alpha-\mathrm{Fe}_{2} \mathrm{O}_{3}$ standard.

TABLE 1: Comparison of Particle Size of Iron Oxide Nanoparticles Using TEM and XRD Analysis

\begin{tabular}{lcc}
\hline & \multicolumn{2}{c}{ particle size $(\mathrm{nm})$} \\
\cline { 2 - 3 } concn of growth soln $(\mathrm{M})$ & TEM analysis & XRD analysis \\
\hline seed & 5.4 & 5.1 \\
0.028 & 6.8 & 6.5 \\
0.058 & 7.6 & 7.2 \\
0.084 & 8.2 & 7.9
\end{tabular}

with the result obtained from the transmission electron microscopy (TEM) analysis.

The weight loss in TG analysis for 5.4, 6.8, and $7.6 \mathrm{~nm}$ iron oxide nanoparticles is shown in Figure 6A. The TGA curve
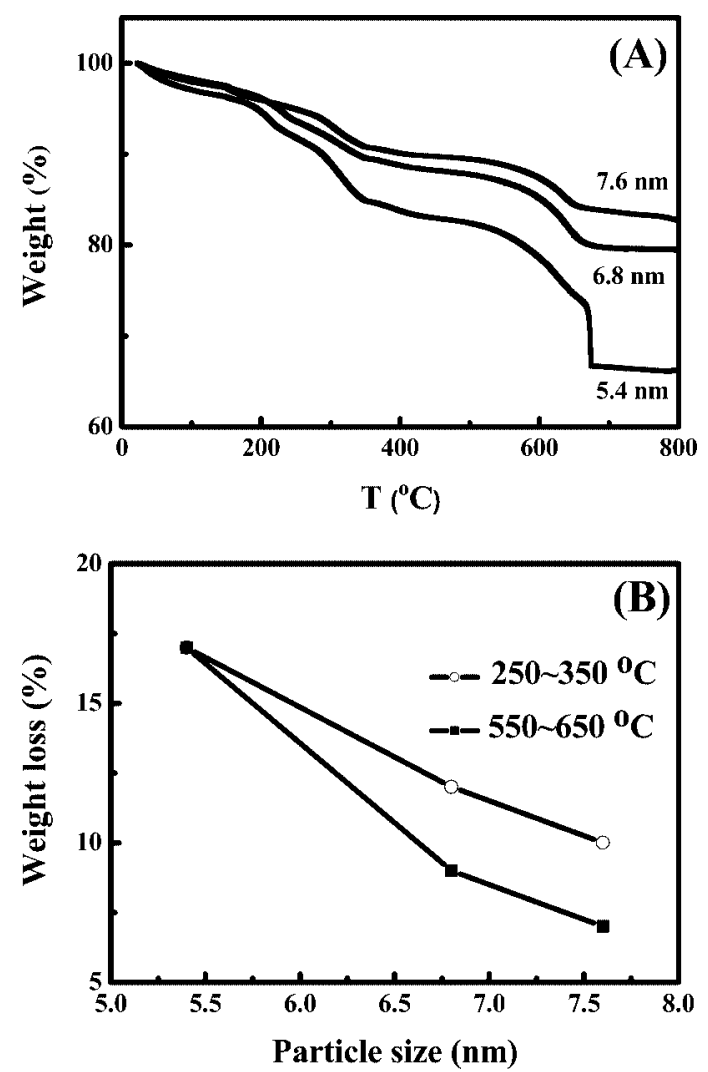

Figure 6. (A) TG analysis for 5.4, 6.8, and $7.6 \mathrm{~nm}$ iron oxide nanoparticles. (B) Analysis of weight loss as a function of particle size at $250 \sim 350$ and $550-650{ }^{\circ} \mathrm{C}$. 

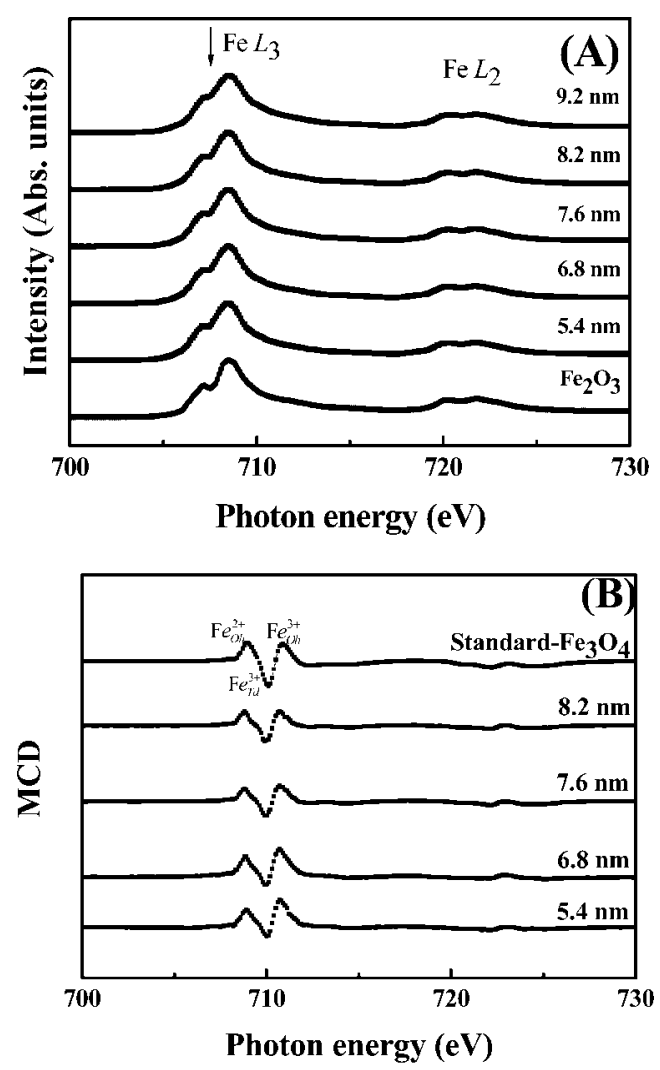

Figure 7. (A) XAS and (B) XMCD spectra for iron oxide nanocrystals with varying particle size.

displays a weight loss from room temperature to $250^{\circ} \mathrm{C}$ and a drop between $250-350{ }^{\circ} \mathrm{C}$ and $550-650{ }^{\circ} \mathrm{C}$, respectively. The weight loss in the low-temperature region results from the decomposition of free oleic acid and the phenyl ether. However, the weight loss at relatively high temperatures can be attributed to the decomposition of oleic acid bound to the nanoparticles. These results suggest the two dissociation regions of oleic acid, owing to the two kinds of interactions between iron oxide nanoparticles and oleic acid. The decomposition at higher temperatures can be attributed to the chemical bonding between iron and the carbonyl group. Since the smaller nanoparticles provide more binding sites on the surface for surfactant than larger ones, the amount of weight loss is related to the particle size due to the higher surface area to volume ratio of nanoparticles (shown in Figure 6B). Thus TG analyses offer important quantitative analysis for different particle sizes of magnetic nanoparticles.

The Fe $\mathrm{L}_{2,3}$-edge XAS and XMCD spectra of iron oxide nanoparticles are shown in Figures 7, panels $\mathrm{A}$ and B, respectively. The XAS spectra of iron oxide nanoparticles with different particle sizes show a peak at around $707 \mathrm{eV}$ that is a characteristic of magnetite. ${ }^{15,16}$ (See the Supporting Information, Figure S1, for the first derivative spectra of XAS analysis.) The shape of the absorption peak is changed with the size of iron oxide nanoparticles, which apparently contributes to the difference in the ratio between $\mathrm{Fe}^{2+}$ and $\mathrm{Fe}^{3+}$ ions in each site of inverse spinel structure. Thus, it is easy to resolve and make a quantitative estimation of the compositions for the iron oxide nanoparticles due to the different orientation of magnetic moments located at different sites using the XMCD technique. 16,17 It is well-known that magnetite possesses an inverse spinel structure consisting of $\mathrm{Fe}^{3+}$ in tetrahedral and $\mathrm{Fe}^{2+}$ and $\mathrm{Fe}^{3+}$ in octahedral sites in one unit cell. The XMCD signal of $\left(\mathrm{Fe}^{3+}\right.$, $\left.\mathrm{Fe}^{2+}\right)_{\mathrm{Oh}}$ and $\mathrm{Fe}^{3+} \mathrm{Td}$ is upward and downward, respectively,
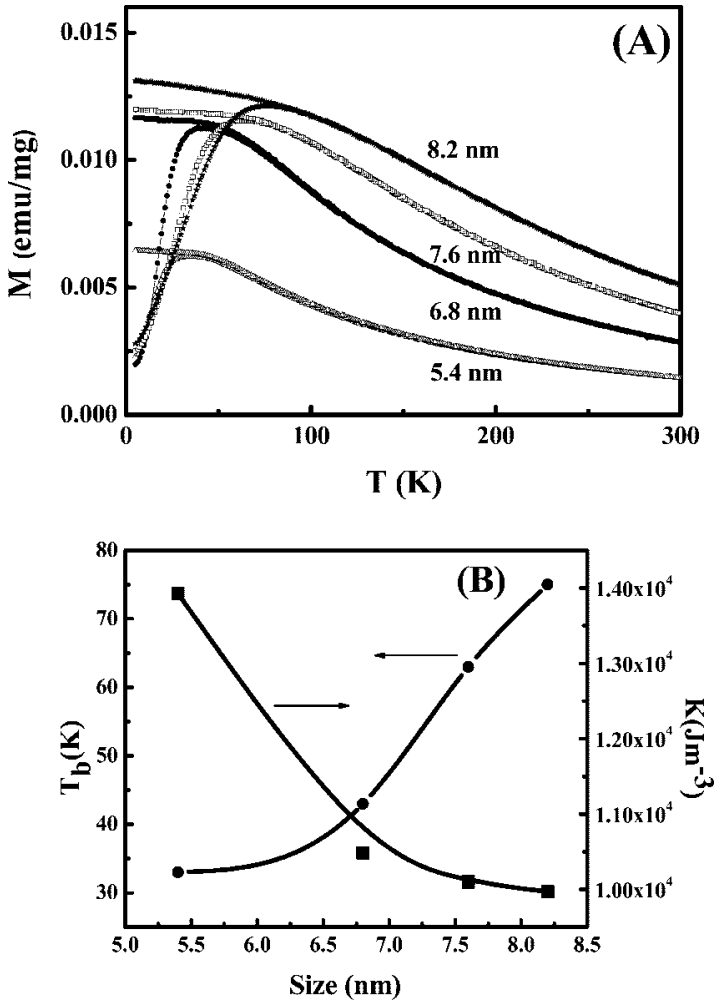

Figure 8. (A) Plot of magnetization versus temperature for iron oxide nanocrystals with varying particle size. (B) Plot of size-dependent blocking temperature $\left(T_{\mathrm{b}}\right)$ and magnetic anisotropic constant $(K)$ for iron oxide nanocrystals with varying particle size.

depending on the change in magnetic field due to the orientation of magnetic moment. ${ }^{31}$ It can be seen from Figure 7B that the signal ratio of $\mathrm{Fe}^{2+}{ }_{\mathrm{Oh}} / \mathrm{Fe}^{3+} \mathrm{Oh}$ for $8.2 \mathrm{~nm}$ nanoparticles is higher than that for other samples. The chemical environment of standard $\mathrm{Fe}_{3} \mathrm{O}_{4}$ is similar to that of the $8.2 \mathrm{~nm}$ nanoparticles, which shows similarity in their oxidation state. The signal ratio of $\mathrm{Fe}^{2+} \mathrm{Oh} / \mathrm{Fe}^{3+} \mathrm{Oh}$ increases as the size increass, hence it was observed that the seeds $(5.4 \mathrm{~nm})$ are at a higher oxidation number than the nanoparticles in the growth solution. The sites on the surface of iron oxide nanoparticles are always occupied by the high oxidation state species. ${ }^{32,33}$ It was observed that the oxidation state is sensitive to the ratio of the specific area of nanoparticles. The average oxidation number increases as the size of the nanoparticles decreases.

The magnetic properties of different sizes of iron oxide nanoparticles were determined by using a SQUID magnetometer. Figure 8A shows the temperature-dependent magnetization measured at 100 Oe from 300 to $10 \mathrm{~K}$. The zero-field cooling (ZFC) curve starts to drop at the blocking temperature $\left(T_{\mathrm{b}}\right)$. It shows the blocking temperatures of $40,50,72$, and 88 $\mathrm{K}$ for the average sizes of $5.4,6.8,7.6$, and $8.2 \mathrm{~nm}$, respectively. Above the blocking temperature, the nanoparticles showed superparamagnetic behavior due to its single magnetic domain nature as evident from the ZFC measurements. For the $5.4 \mathrm{~nm}$ particles, the observed blocking temperature is $40 \mathrm{~K}$, which is close to the lowest blocking temperature measured. With increasing particle size, the blocking temperature increases as shown in Figure 8B. The magnetic anisotropy constant $K$ was calculated by using the following equation: ${ }^{34-36}$

$$
K=25 K_{\mathrm{B}} T_{\mathrm{b}} / V
$$

where $K_{\mathrm{B}}$ is the Boltzman constant, $T_{\mathrm{b}}$ is the blocking temperature, and $V$ is the volume of one nanoparticle determined by 
TABLE 2: Table of $T_{\max }, T_{\text {splitting, }}$ and $\Delta T$ Calculated Using SQUID Analysis

\begin{tabular}{cccc}
\hline av diameter $(\mathrm{nm})$ & $T_{\mathrm{b}}(\mathrm{K})$ & $T_{\text {splitting }}(\mathrm{K})$ & $\Delta T(\mathrm{~K})$ \\
\hline 5.4 & 33 & 40 & 7 \\
6.8 & 43 & 50 & 7 \\
7.6 & 63 & 72 & 9 \\
8.2 & 75 & 88 & 13
\end{tabular}

TEM measurements. Generally, the thermal energy induces the magnetic spin disorderedness so that the magnetization of particles increases with the decreasing temperature. However, below the blocking temperature, the magnetization suddenly drops due to the magnetic anisotropic effect and the interaction between nanoparticles. ${ }^{37-40}$ Hence, the blocking temperature point indicates the key parameter for magnetic materials, which is the result of competition between the magnetic anisotropic energy and the thermal energy. At the nanoscale level, the magnetic spin on the particle surface is influenced easily by the distortion of coordination. ${ }^{12}$ Hence the nanoparticles with large a surface area to volume ratio have low magnetization and a high anisotropy constant. This displays the splitting point ( $T_{\text {splitting }}$, which is the cleavage value of the ZFC and FZ curve, and the maximum point $\left(\mathrm{T}_{\mathrm{b}}\right)$, which is the maximum value in the ZFC curve (shown in Table 2). Since $T_{\text {splitting }}$ and $T_{\max }$ depend on the distribution of anisotropy energy in large size and major size, the deviation $(\Delta T)$ between two points reflects the degree of monodispersity or size distribution (or standard deviation). ${ }^{41,42}$ The measurements carried out by SQUID are consistent with the standard deviation calculated by TEM images.

For the biomedical applications of iron oxide nanoparticles such as magnetic resonance imaging, ${ }^{8}$ it is necessary to modify the hydrophilic ligand on the nanoparticles surface to promote the suspension of nanoparticles in aqueous media. In the present studies, we have modified the nanoparticles surface by replacing the oleate species using the tetramethylammonium 11-aminoundecanoate ligand to promote hydrophilicity. ${ }^{14}$ The carboxylate group of the ligand binds to the surface iron and exposes the hydrophilic amino group to aqueous media. For analyzing the biocompatibility, the hydrophilic nanoparticles of size 5.4 and $7.6 \mathrm{~nm}$ in the concentrations range of $0.1,1,10$, and $100 \mu \mathrm{M}$ were treated with the normal breast epithelial cells (H184B5F5/ M10) and the three types of breast cancer cells (SKBR3, MB157, and T47D) for $72 \mathrm{~h}$. Figure 9 shows the cell viability results for normal as well as breast cancer cells with 5.4 and $7.4 \mathrm{~nm}$ nanoparticles. The cytotoxicity studies revealed that there is no obvious change in cell viability in the studied concentration range of magnetic nanoparticles, although the $100 \mu \mathrm{M}$ nanoparticle concentration is considered to be far higher than the normal use. Thus, the as-prepared nanoparticles showed biocompatibility and nontoxicity against the normal as well as cancerous cell lines. ${ }^{43}$

\section{Conclusions}

In summary, we have synthesized the iron oxide nanoparticles with monodispereity using the seed-mediated method combined with thermal decomposition. The size evolution has been demonstrated by understanding the growth process for the formation of monodispersed nanoparticles. It was observed that the monodispersed nanoparticles were formed by the intraparticle ripening process, and the primary nucleation of the particles in the growth solution played an important role in establishing the buffer condition to prevent the seed from undergoing random growth. It is also possible to control the distribution and size
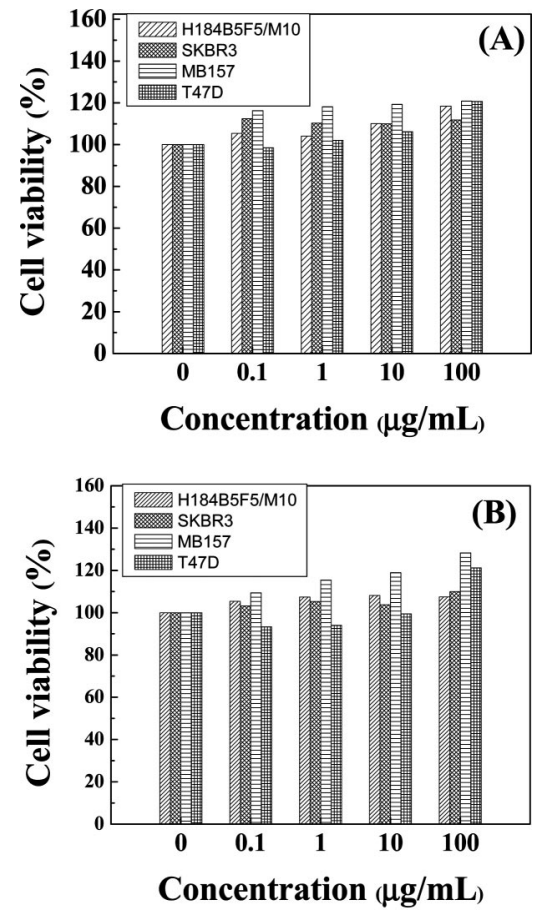

Figure 9. The cytotoxicity tests for breast epithelial cell (H184B5F5/ M10) and the three types of breast cancer cells, using (A) 5.4 and (B) $7.4 \mathrm{~nm}$ iron oxide nanoparticles.

of the particles by tuning the temperature and the concentration of growth solution. Under the high concentration condition, the nucleation and growth process compete with each other to consume the monomers, whereas the temperature can affect the degree of dissociation of precursor for the further growth of nanoparticles. Hence, we modified these factors to obtain the monodispersed iron oxide nanoparticles with standard deviation $(\sigma<1)$. The magnetic nanoparticles showed well-defined superparamagnetic and blocking temperature due to the size effects consistent with the TEM images. The TG analyses showed that the surfaces of nanoparticles were occupied by different numbers of ligands via physical absorption and chemical binding, which changes with particle size, owing to the surface area to volume ratio. The as-prepared iron oxide nanoparticles showed biocompatibility and nontoxicity against the normal as well as cancerous cell lines.

Acknowledgment. The authors would like thank the National Science Council of Taiwan for financially supporting this research under Contract Nos. NSC 96-2120-M-002-019, NSC 96-2120-M-002-017, and NSC 96-2120-M-001-009.

Supporting Information Available: First derivative of XAS spectra, the TEM image of iron oxide nanoparticles in aqueous media after replacing the oleate species by the tetramethylammonium 11-aminoundecanoate ligand, and its histogram of size distribution. This material is available free of charge via the Internet at http://pubs.acs.org.

\section{References and Notes}

(1) Song, H. T.; Choi, J. S.; Huh, Y. M.; Kim, S.; Jun, Y. W.; Suh, J. S.; Cheon, J. J. Am. Chem. Soc. 2005, 127, 9992.

(2) Jun, Y. W.; Huh, Y. M.; Choi, J. S.; Lee, J. H.; Song, H. T.; Kim, S. J.; Yoon, S.; Kim, K. S.; Shin, J. S.; Suh, J. S.; Cheon, J. J. Am. Chem. Soc. 2005, 127, 5732 .

(3) Weizmann, Y.; Patolsky, F.; Katz, E.; Willner, I. J. Am. Chem. Soc. 2003, 125, 3452 .

(4) Wan, J.; Cai, W.; Meng, X.; Liu, E. Chem. Commun. 2007, 5004. 
(5) Bao, J.; Chen, W.; Liu, T.; Zhu, Y.; Jin, P.; Wang, L.; Liu, J.; Wei, Y.; Li, Y. ACS Nano 2007, 1, 293.

(6) Kim, J.; Lee, J. E.; Lee, J.; Yu, J. H.; Kim, B. C.; An, K.; Hwang, Y.; Shin, C. H.; Park, J. G.; Kim, J.; Hyeon, T. J. Am. Chem. Soc. 2006, 128,688 .

(7) Kim, J.; Lee, J. E.; Lee, S. H.; Yu, J. H.; Lee, J. H.; Park, T. G.; Hyeon, T. Adv. Mater. 2008, 20, 478.

(8) Gass, J.; Poddar, P.; Almand, J.; Srinath, S.; Srikanth, H. Adv. Funct. Mater. 2006, 16, 71.

(9) Salgueiriño-Maceira, V.; Correa-Duarte, M. A.; Spasova, M.; LizMarzán, L. M.; Farle, M. Adv. Funct. Mater. 2006, 16, 509.

(10) Daou, T. J.; Pourroy, G.; Bégin-Colin, S.; Grenèche, J. M.; UlhaqBouillet, C.; Legaré, P.; Bernhardt, P.; Leuvrey, C.; Rogez, G. Chem. Mater. 2006, 18, 4399.

(11) Wang, Z.; Guo, H.; Yu, Y.; He, N. J. Magn. Magn. Mater. 2006, 302, 397.

(12) Woo, K.; Hong, J.; Choi, S.; Lee, H.-W.; Ahn, J.-P.; Kim, C. S.; Lee, S. W. Chem. Mater. 2004, 16, 2814.

(13) Hyeon, T.; Lee, S. S.; Park, J.; Chung, Y.; Na, H. B. J. Am. Chem. Soc. 2001, 123, 12798.

(14) Sun, S.; Zeng, H.; Robinson, D. B.; Raoux, S.; Rice, P. M.; Wang,

S. X.; Li, G. J. Am. Chem. Soc. 2004, 126, 273.

(15) Song, H.-T.; Choi, J.-S.; Huh, Y.-M.; Kim, S.; Jun, Y.-W.; Suh,

J.-S.; Cheon, J. J. Am. Chem. Soc. 2004, 126, 273.

(16) Park, J.; Lee, E.; Hwang, N.-M.; Kang, M.; Kim, S. C.; Hwang, Y.; Park, J.-G.; Noh, H.-J.; Kim, J.-Y.; Park, J.-H.; Hyeon, T. Angew. Chem., Int. Ed. 2005, 44, 2872.

(17) Park, J.; An, K.; Hwang, Y.; Park, J.-G.; Noh, H.-J.; Kim, J.-Y.;

Park, J.-H.; Hwang, N.-M.; Hyeng, T. Nat. Mater. 2004, 3, 891.

(18) Kwon, S. G.; Piao, Y.; Park, J.; Angappane, S.; Jo, Y.; Hwang,

N. M.; Park, J. G.; Hyeon, T. J. Am. Chem. Soc. 2007, 129, 12571.

(19) Park, J.; Joo, J.; Kwon, S. G.; Jang, Y.; Hyeon, T. Angew. Chem., Int. Ed. 2007, 46, 4630.

(20) Kok, S. H. L.; Chui, C. H.; Lam, W. S.; Chen, J.; Lau, F. Y.; Cheng, G. Y. M.; Wong, R. S. M.; Lai, P. P. S.; Leung, T. W. T.; Tang, J. C. O.; Chan, A. S. C. Int. J. Mol. Med. 2006, 17, 945.

(21) Sasaki, E.; Tominaga, K.; Kuwamura, H.; Watanabe, T.; Fujiwara, Y.; Oshitani, N.; Higuchi, K.; Arakawa, T. J. Gastroenterol. 2007, 42, 816. (22) Chui, C. H.; Cheng, G. Y. M.; Ke, B.; Lau, F. Y.; Wong, R. S. M.; Kok, S. H. L.; Fatima, S.; Cheung, F.; Cheng, C. H.; Chan, A. S. C.; Tang, J. C. O. Int. J. Mol. Med. 2004, 14, 925.
(23) Cheung, F.; Chui, C. H.; Chan, A. S. C.; Lau, F. Y.; Cheng, G. Y. M.; Wong, R. S. M.; Kok, S. H. L.; Teo, I. T. N.; Cheng, C. H.; Tang, J. C. O. Int. J. Mol. Med. 2005, 16, 925.

(24) Mullin, J. W. Crystallization, 3rd ed.; Butterworth-Heinemann: Boston, MA, 1997.

(25) Talapin, D. V.; Rogach, A. L.; Haase, M.; Weller, H. J. Phys. Chem. B 2001, 105, 12278 .

(26) Xiaogang, P.; Wickham, J.; Alivisatos, A. P. J. Am. Chem. Soc. 1998, 120, 5343 .

(27) Chen, Y.; Kim, M.; Lian, G.; Johnson, M. B.; Peng, X. J. Am. Chem. Soc. 2005, 127, 13331.

(28) Smet, Y. D.; Deriemaeker, L.; Finsy, R. Langmuir 1999, 15, 6745.

(29) Chen, Y.; Johnson, E.; Peng, X. J. Am. Chem. Soc. 2007, 129, 10937.

(30) Sugimoto, T. Adv. Colloid Interfac. Sci. 1987, 28, 65.

(31) Stohr, J. J. Magn. Magn. Mater. 1999, 200, 470.

(32) Vanheusden, K.; Warren, W. L.; Seager, C. H.; Tallant, D. R.; Voigt, J. A. J. Appl. Phys. 1996, 79, 7983. 174.

(33) Peng, Y.-Y.; Hsieh, T.-E.; Hsu, C.-H. Nanotechnology 2006, 17,

(34) Rancourt, D. G.; Daniels, J. M. Phys. Rev. B 1984, 29, 2410.

(35) Bødker, F.; Mørup, S.; Linderoth, S. Phys. Rev. Lett. 1994, 72 , 282.

(36) Luo, W.; Nagel, S. R.; Rosenbaum, T. F.; Rosenbaum, R. E. Phys. Rev. Lett. 1991, 67, 2721.

(37) Mørup, S. Phys. Rev. Lett. 1994, 72, 3278.

(38) Morales, M. P.; Veintemillas-Verdaguer, S.; Montero, M. I.; Serna, C. J. Chem. Mater. 1999, 11, 3058.

(39) Bodker, F.; Morup, S.; Linderoth, S. Phys. Rev. Lett. 1994, 72, 282.

(40) Bae, C. J.; Angappane, S.; Park, J. G.; Lee, Y.; Lee, J.; An, K.; Hyeon, T. Appl. Phys. Lett. 2007, 91, 102502.

(41) Ma, D.; Veres, T.; Clime, L.; Normandin, F.; Guan, J.; Kingston, D.; Simard, B. J. Phys. Chem. C 2007, 111, 1999.

(42) Ayoub, N. Y. Jpn. J. Appl. Phys. 1991, 30, 3381.

(43) Lee, Y. K.; Kim, D. H.; Lee, S. H.; Im, K. H.; Kim, K. N.; Kim, K. M.; Shim, I. B.; Lee, M. H. Curr. Appl. Phys. 2006, 6, e242.

JP803452J 